\title{
A Guide for Choosing the Right Calibration for Torque Transducers
}

\author{
Wegener, Georg / Stenner, Lioba \\ Hottinger Baldwin Messtechnik GmbH \\ Im Tiefen See 45 \\ 64293 Darmstadt
}

\section{Introduction}

Torque transducers (e.g. torque shafts, torque flanges) have become increasingly important during the last decades. Measuring torque is crucial for testing the performance of a wide range of rotating machinery. Some important examples are engine and transmission test stands in the automotive industry, testing of pumps or electric motors and many other mechanisms like steering gear. Another field of torque measurement is in torque tools used in assembly, from simple torque wrenches to rotary assembly tools (either electric or pneumatic). However the latter will not be covered by the present paper.

Basically, torque transducers like any other test and measurement equipment used in industry needs to be calibrated regularly. Traceable calibration for all testing equipment is a requirement of every serious quality management system. It is essential for meeting any standard of the ISO 9000 family. In some branches of industry, the requirements for a traceable calibration are even stricter. An example is ISO/TS 16949 used by the European and American automotive industry.

But even though undoubtedly in many cases a calibration is required, it remains open, which kind of calibration to choose in view of the variety of different types of calibrations for torque transducers. The most obvious criterion is the difference between accredited calibration and what is called a working standard calibration. In Germany accreditation for calibration laboratories is done by the DKD ("Deutscher Kalibrierdienst" = German Calibration Service). Similar institutions exist in all major industrial countries, e.g. COFRAC in France, UKAS in the United Kingdom and SIT in Italy.

The difference between an accredited and a working standard calibration is primarily a formal one, but there are also big technical differences. Usually, the calibration procedures (loading sequences) and the evaluation procedures are much more elaborate in the case of accredited calibrations. But the high metrological quality is also expensive. Therefore it is the aim of this contribution to define criteria which help users in their decision for a specific type of torque calibration. It will also show that a compromise which is quite attractive for many fields of application exists.

\section{Calibration methods for torque transducers}

By and large, equipment and machines for calibrating torque transducers can be subdivided according to the reference standard used. In lever-arm-mass systems a precisely defined torque is generated when the weight force of calibrated masses acts on the test specimen by means of a lever arm of known length. This is the technology currently used in all calibration laboratories for the high and highest grades of accuracy.

The second principle is to use a torque reference transducer to provide the reference torque. In principle, systems with torque reference transducers can use any mechanism to generate the torque. The generated torque is then measured with the aid of a torque reference transducer. Features of both principles are combined in calibration machines with a force reference transducer and a lever arm.

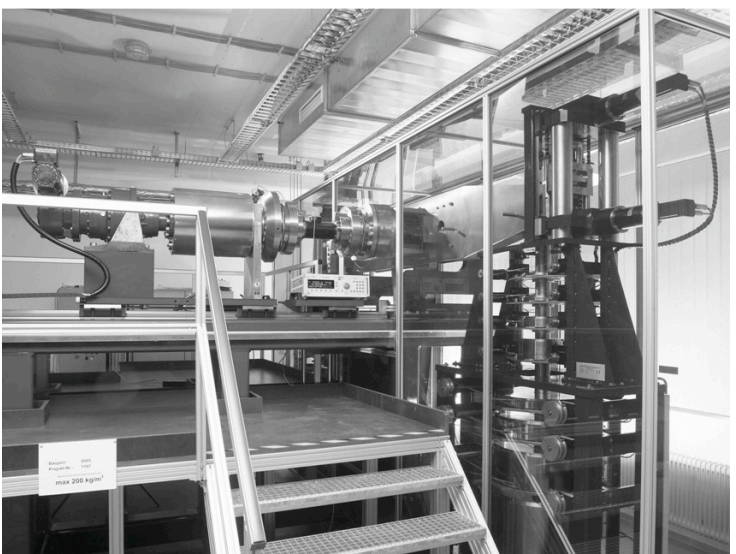

Fig. 1: $25 \mathrm{kN} \cdot \mathrm{m}$ torque calibration machine of HBM Germany.

Working principle: lever arm and masses. Measurement uncertainty: $0.008 \%$ of actual value 


\section{Traceability chain and orders of rank in calibration}

\subsection{Overview / the calibration pyramid} The purpose of calibration is to provide traceability of the measuring instrument (here: a torque transducer) to national standards. This means that there must be an unbroken chain of comparisons to stated references to the national standard for the measurand underconsideration. National standards for torque are the torque calibration machines with the highest rank. They are also referred to as "torque standard machines". They are owned by the National Measurement Institutes (NMIs) like PTB in Germany, LNE in France, the NPL in the UK and INRIM in Italy.

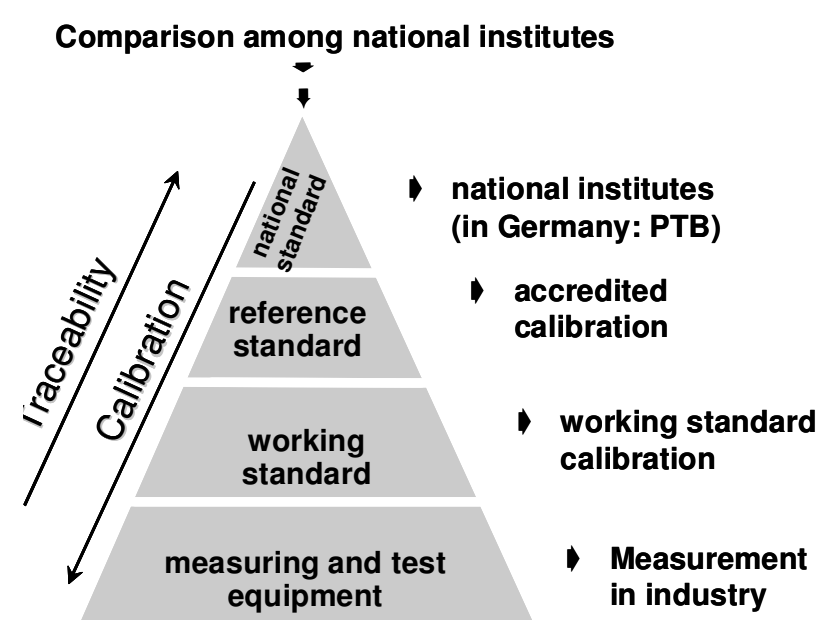

Fig. 2: $\quad$ The calibration pyramid

3.2. Accredited calibration laboratories (e.g. in Germany: DKD laboratories)

Calibration by an accredited laboratory applying accredited procedures is second best after calibration by the National Metrology Institutes themselves. Usually the procedure is following an approved standard or regulation. Most common are the German DIN standard DIN 51309 [1] or the European EA-10/14 (lately renamed to EURAMET/cg-14/v.01) [2].

Such calibration certificates have a very high rank as a documentation of traceability since the calibration laboratory itself has been certified by the official accreditation body. One of the most active accreditation bodies in the field of torque calibration is the German DKD. When the DKD started activities in torque in 1990, the first accredited laboratory was the calibration laboratory of $\mathrm{HBM} \mathrm{GmbH}$, a manufacturer of measurement transducers and instrumentation, torque transducers among them. Accreditation for calibration laboratories follows the international standard ISO 17025 [3]. The accreditation provides a proven standard of management and documentation of traceability of their reference standards.

\subsection{Working standard calibration}

Since calibrations according to the regulations used for an accredited certificate are expensive, most industrial users of torque transducers prefer the working standard calibration. Compared with DKD calibrations, working standard calibrations are usually carried out more simply and are therefore less costly to perform. They can be carried out not only by DKD accredited laboratories, but also by suppliers whithout accreditation or by the user of the torque transducers. The responsibility for determining the procedures and evaluatin algorithms af the process rests with the laboratory performing the calibration.

Users who order a working standard calibration from accredited calibration laboratories like HBM calibration laboratory in Darmstadt, Germany usually benefit from the experience and structures of the accredited laboratory.

\section{Calibration and evaluation procedures in the different orders of rank}

\subsection{Accredited calibration according to "classical" standards and guidelines}

The type of calibration with the maximum metrological information is certainly the comprehensive calibration within the framework of an accredited laboratory applying internationally accepted standards, in Germany for example, this would be a classical DKD calibration.

A very important feature of accredited calibration is a dependable computation of the uncertainty of measurement for each individual value given in the certificate. Another help offered by such a calibration for assessing the calibrated transducer is the classification. Classification is carried out on the basis of the metrological parameters obtained and determined during calibration. For each class, there are specified upper bounds for each parameter. Since the parameters are computed for each calibrated torque step individually, classification too can be different for the different steps. 


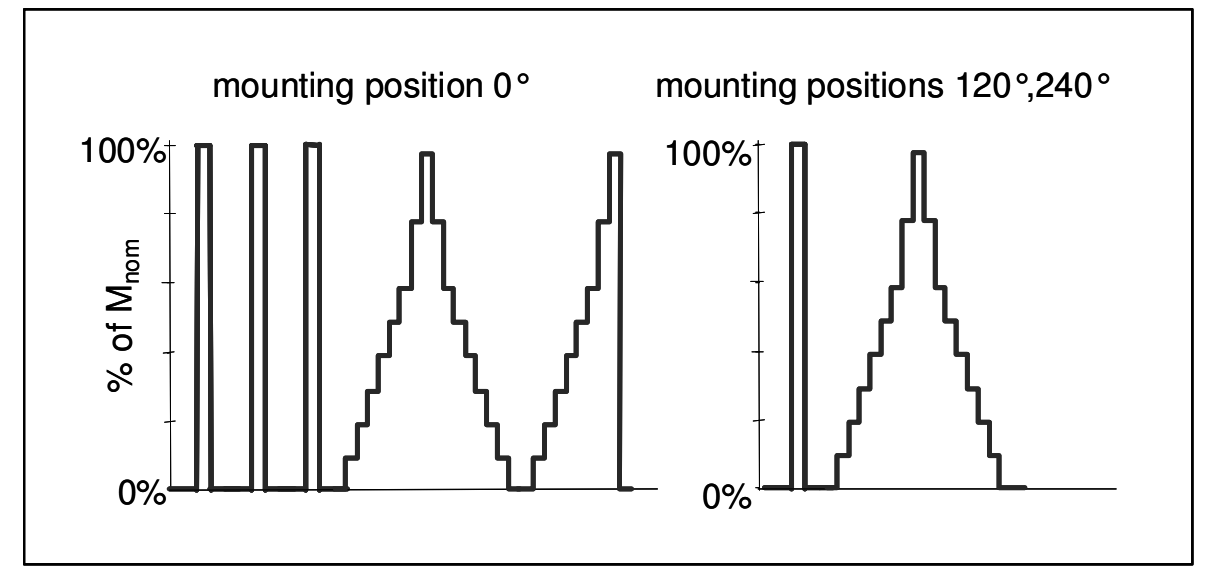

Fig. 3: Loading sequence of an 8-step torque calibration according DIN 51309

One of the main features of all the calibration procedures in this group is the fact that the calibration is carried out in three different mounting positions. This means that the transducer is dismounted from the calibration machine, rotated around its torque axis by $120^{\circ}$ and then re-mounted. Comparing the measurement results of the same torque step after this kind of re-mounting allows to determine a parameter called reproducibility. This parameter is very important for estimating the deviation of the torque transducer's characteristic between one individual measurement taken during calibration and its subsequent use. Therefore calibration experts agree on the fact, that it is not possible to calculate an individual measurement uncertainty without knowing the reproducibility.

The second special feature of the load sequence of all these calibrations is the repeat measurement: In the first mounting position, there is a second loading sequence with incremental load. Comparing the measurement results of the same torque step for the two subsequent incremental load sequences in the same mounting position allows to determine a parameter known as repeatability. This parameter describes the statistical spread. It is also required for a correct computation of the individual measurement uncertainty.

If a torque transducer is supposed to be used under alternating torque operating conditions, the German DKD has provided a special guideline for an alternating torque calibration. This standard, the DKD regulation DKD-R 3-5 [4] is an extension based on the DIN 51309.

\subsection{Working standard calibration}

The loading sequence and evaluation procedure of working standard calibrations may differ among calibration laboratories due to the fact that there are no generally binding regulations or standards.

A typical loading sequence of a simple working standard calibration has one to three pre-load cycles followed by the main loading sequence: the torque load is increased stepwise (incremental series) and decreased afterwards (decremental series). Such a procedure allows to determine the relation between physical load and output signal of a torque transducer. In this paper such a relation is referred to as "characteristic" regardless whether it is expressed by a sensitivity, a best fit equation or a tabular allocation.

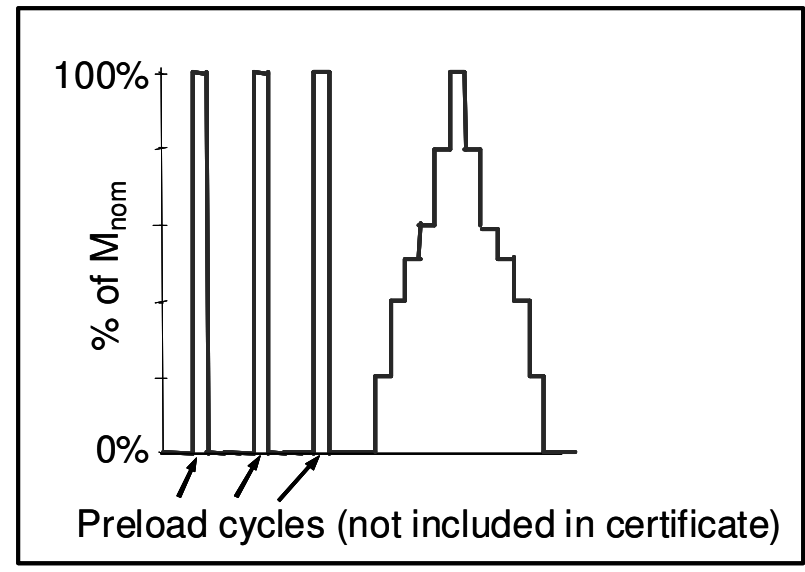

Fig. 4: Typical loading sequence for a torque working standard calibration (6-steps, incremental and decremental). 
Most working standard calibrations do not contain a computation of the uncertainty of measurement. This is a significant disadvantage for two reasons: First, such a calibration result is only of limited value for assessing the measurement uncertainty of the processes in which the transducer is used subsequent to calibration. Second, their value as a document of traceability is limited. In the past few years, auditors tend to a more strict definition of traceability. According to this definition, it is not sufficient to know the measurement uncertainty of the equipment used for the calibration, it is necessary to have a measurement uncertainty determined for the individual calibration.

\subsection{Simplified accredited calibration according to VDI/VDE 2646}

In 2005 a new calibration guideline in Germany was released, the VDI/VDE guideline 2646 [5]. The aim of this guideline was to provide a general guideline for calibrations with medium requirements of accuracy. In contrast with all existing procedures for working standard calibrations, this guideline includes the calculation of an individual uncertainty of measurement.

The loading sequence includes a repeat series, but no reproducibility measurements (measurements in different mounting positions). Nevertheless, the parameter for reproducibility is required for the uncertainty calculation. The guideline permits to use a reproducibility parameter gained from experience or statistical information about

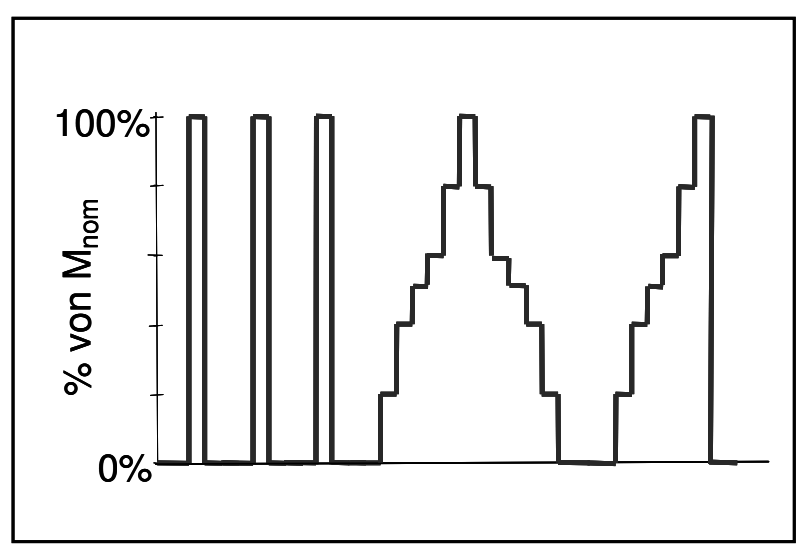

Fig. 5: Loading sequence for a torque calibration according to VDI/VDE 2646 (6-steps).

the type of torque transducer. However it allows only a measurement uncertainty of $0.5 \%$ or above, unless the experience-based reproducibility parameter is derived from a documented statistical method. Some German calibration laboratories have implemented working standard calibrations according to this guideline.

The HBM calibration laboratory in Darmstadt has chosen a different method. This laboratory has established a database with calibration data of hundreds of torque transducers and defined a wellfounded statistical method. This method has been approved by the accreditation board of the German Calibration Service DKD. Now HBM can offer calibrations according to guideline VDI/VDE 2646 as DKD calibrations (accredited calibrations). The method was presented on the international IMEKO (International Measurement Confederation) conference in 2006 [7]. However such statistical information is not available for all models of torque transducers and not for each special type of calibration. Namely it is limited to torque transducers currently manufactured by HBM and to calibrations in the full nominal load range of the respective transducer type.

Since it is aimed at a different application field than the classical accredited calibration, the certificate does not contain every individual parameter (e.g. hysteresis, deviation from the best fit curve, ...) for each load step. Instead, HBM decided to include an overview of the most relevant parameters, like the maximum of hysteresis and linearity deviation in the calibration range. A very useful parameter for the practitioner is the parameter called "optimised sensitivity". This parameter enables to adjust a common measuring amplifier according to the best fit line for the transducer's characteristic (determined by a least squares algorithm). Another extra is the information about the mechanical remanence. This parameter is contained on each calibration certificate which is for both clockwise and counter-clockwise torque. So there is no longer the confusing distinction between a calibration for both load senses and the calibration for alternating torque. All these extra parameters go beyond the scope of the calibration certificate defined by the VDI/VDE guideline. Nevertheless their definitions are not HBM specific. They are conform with the VDI 2639 standard "Characteristics of Torque Transducers" [6]. 
The DKD calibration according to the VDI guideline can be called a simplified DKD calibration and offers multiple benefit for the customer:

- the price is as low as for a working standard calibration

- high rank as a certificate of traceability that can be attained only by accredited calibrations

- calculation of the individual uncertainty of measurement

- several relevant parameters for assessing the accuracy of the calibrated torque transducer in practical use

- statement of conformity (okay / not okay statement) with respect to the most relevant datasheet specifications

\section{Requirements of different application fields and the adequate types of calibration}

Of course the comprehensive calibration according to a standard like DIN 51309 [1] covers all the needs of most applications. However, the cost of such a calibration is significant and so it has to be considered for which cases this costly procedure is really necessary.

\subsection{Torque transducers used as calibration equipment}

For torque transducers used as calibration equipment (e.g. reference or transfer transducers in calibration laboratories or transducers being part of equipment for calibrating test stands on site) a highly qualified traceability is most important. Therefore the classical accredited (DKD) calibration is usually the right choice. Due to the small number of references even a higher price is not causing big overall costs.

\subsection{Torque transducers used in test stands}

In test stands for certification tests the requirements concerning accuracy are usually also relatively high. The requirements concerning traceability are extremely high. For some pollution certifications, a re-calibration has to be performed before each certification test.

In test stands in $R \& D$ the requirements in accuracy of the torque measuring equipment are also relatively high. Often the user can benefit if particular metrological properties are documented in the calibration certificate. An example is the "mechanical remanence" (also referred to as hysteresis of the zero point or toggle effect). This effect quantifies the additional measurement

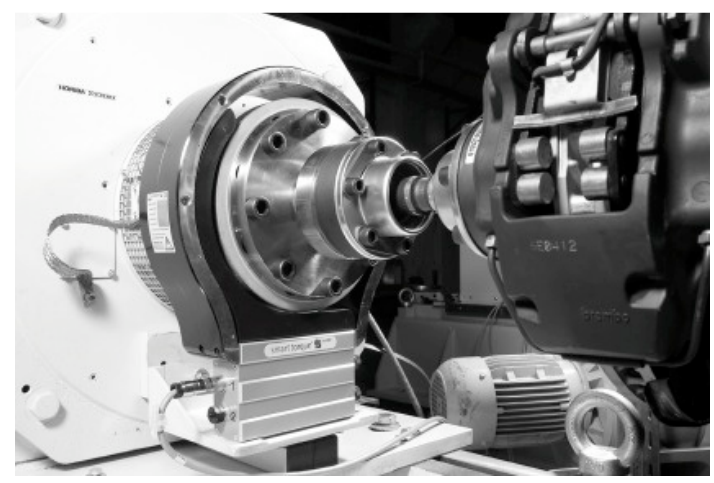

Fig. 6: Torque transducer (T12 by HBM) in an automotive test stand. deviation occuring with alternate torque, the recurring change between clockwise and counterclockwise torque.

Costs for the calibration may be significant due to the bigger number of transducers (e.g. 5 torque transducers for one transmission test stand for 4-wheel drive cars). For test stands in end of line testing the accuracy requirements are usually only average. The traceability requirements depend on the quality policy of manufacturer.

Concluding from the above combination of high, but not extremely high demands and the cost, the best choice for torque transducers in test stand applications is the simplified, yet accredited calibration. 


\begin{tabular}{|c|c|c|c|c|c|c|c|}
\hline & \multirow[b]{2}{*}{$\begin{array}{l}\text { standard / } \\
\text { guideline }\end{array}$} & \multirow[b]{2}{*}{ 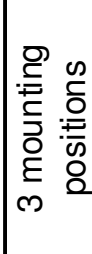 } & \multirow[b]{2}{*}{ 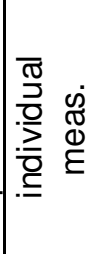 } & \multirow[b]{2}{*}{ 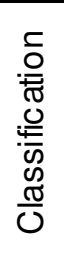 } & \multicolumn{3}{|c|}{ additional info } \\
\hline & & & & & 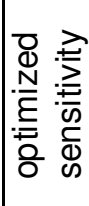 & 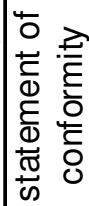 & 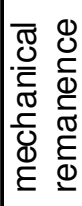 \\
\hline \multirow{3}{*}{$\begin{array}{l}\text { "classical" accredited } \\
\text { calibration }\end{array}$} & EURAMET & $x$ & $x$ & $x$ & 一 & - & - \\
\hline & DIN 51309 & $x$ & $x$ & $x$ & 一 & - & - \\
\hline & DKD-R 3-5 )* & $x$ & $x$ & $x$ & 一 & - & $x$ \\
\hline $\begin{array}{l}\text { simplified accredited } \\
\text { calibration }\end{array}$ & $\begin{array}{l}\text { VDI/VDE } \\
2646)^{\star *}\end{array}$ & - & $x$ & $x$ & $x$ & $x$ & $x$ \\
\hline $\begin{array}{l}\text { enhanced working standard } \\
\text { calibration }\end{array}$ & $\begin{array}{l}\text { VDI/VDE } \\
2646\end{array}$ & - & $x$ & - & 一 & 一 & 一 \\
\hline $\begin{array}{l}\text { "classical" working } \\
\text { standard calibration }\end{array}$ & $\begin{array}{c}\text { no general } \\
\text { standard }\end{array}$ & - & 一 & - & 一 & 一 & 一 \\
\hline
\end{tabular}

)$^{*} \quad$ for alternating torque only

)$^{* *}$ only available at HBM GmbH, Darmstadt, Germany

Table 1: Overview of information content provided by different torque calibration types

\section{References}

[1] DIN (German Standards Institute), DIN 51309, Werkstoffprüfmaschinen - Kalibrierung von Drehmomentmessgeräten für statische Drehmomente (Materials Testing Machines - Calibration of static torque measuring devices), 2005.

[2] EURAMET (European Association of National Metrology Institutes), EURAMET/cg-14/v.01 Guidelines on the Calibration of Static Torque Measuring Devices, 2007. (Previously EA-10/14)

[3] ISO (International Organization for Standardization) and others, DIN EN ISO/IEC 17025, Allgemeine Anforderungen an die Kompetnz von Prüf- und Kalibrierlaboratorien / General requirements for the competence of testing and calibration laboratories (German and English version EN ISO/IEC 17025:2005), 2005.

[4] DKD (German Calibration Service), Guideline DKD R 3-5 Kalibrierung von Drehmomentmessgeräten für statische Wechseldrehmomente (Calibration of Torque Measuring Devices for Static Alternating Torque), 2002.

[5] VDI/VDE (Society of German Engineers), Guideline VDI/VDE 2646, Drehmomentmessgeräte Mindestanforderungen an Kalibrierungen (Torque measuring devices - Minimum requirements in calibrations), 2006.

[6] VDI/VDE (Society of German Engineers), Guideline VDI/VDE 2639, Kenngrößen für Drehmomentaufnehmer / Characteristics of Torque Transducers (bi-lingual German / English), 2006.

[7] Wegener, Georg; Werner, Hans-W., Suggestions on how to include the effect of reproducibility in the evaluation of simplified calibration procedures, Paper presented at the IMEKO 20th TC3, 3rd TC16 \& 1st TC22 International Conference, November 27th to December 1st, 2007, Merida, Mexico. 